Multiple sclerosis, emotion perception and social functioning.

Short title: MS, emotion perception and social function.

Bogna Radlak ${ }^{1,2}$, Clare Cooper ${ }^{3}$, Fiona Summers ${ }^{4}$ \& Louise H. Phillips ${ }^{1}$ *

1: School of Psychology, University of Aberdeen

2: Department of Neuropsychology, Ninewells Hospital in Dundee

3: Health Psychology Group, University of Aberdeen

4: Department of Neuropsychology, Aberdeen Royal Infirmary

* Corresponding author. Louise H. Phillips, School of Psychology, University of Aberdeen, Aberdeen, Scotland, UK, AB24 3FX. Email louise.phillips@abdn.ac.uk. ORCID: 0000-0002-0988-3028 


\begin{abstract}
People with Multiple sclerosis (MS) can experience problems in interpreting others' emotions from faces or voices. However, to date little is known about whether difficulties in emotion perception in MS are related to broader aspects of social functioning. Also, there are few studies reporting the effect of MS on more ecologically valid assessments of emotion perception using multimodal videos. The current study looks at (i) the effect of MS on perceiving emotions from faces, voices and multimodal videos; (ii) the possible role of slowed processing and executive dysfunction in emotion perception problems in MS, and (iii) the relationship between emotion perception and broader social functioning in MS. 53 people with MS and 31 healthy controls completed tasks of emotion perception and cognition, and assessed their levels of social support and social participation. Participants with MS performed worse than demographically-matched controls on all measures of emotion perception. Emotion perception performance was related to cognitive measures in those with MS. Also, significant associations were found between emotion perception difficulties in MS and poorer social function. In particular, people with MS who had poorer emotion perception also reported lower levels of social support from their friends, and regression analysis showed that this prediction was maintained even when disease severity and cognitive function were taken into account. These results show that problems with emotion perception in MS extend to more realistic tasks, and may predict key aspects of social functioning.
\end{abstract}

Keywords: multiple sclerosis, emotion perception, social function, social cognition. 


\section{Multiple sclerosis, emotion perception and social functioning.}

Multiple sclerosis (MS) is the most common cause of neurological disability in young adults (Compston \& Coles, 2002). MS often restricts social functioning: for instance Einarsson et al. (2006) found that $65 \%$ of their MS sample were restricted in social activities. While the challenging physical symptoms of the disease will restrict social engagement for some people with MS (pwMS), another factor which might be important is impairment in the social cognitive skills needed to understand others' emotional states.

Emotion perception is the ability to recognise affective content from cues such as auditory tone, facial expressions and bodily movements (Bornhofen \& McDonald, 2008). Accurate reading of others' emotions is necessary for successful social interactions (McDonald et al., 2004). Meta-analyses indicate that multiple sclerosis (MS) causes reliably poorer performance on emotion perception with moderate effect size $(d=0.61$, Bora, Özakbaş, Velakoulis \& Walterfang, 2016; $g=0.64$, Cotter et al., 2016), even at the early stages of MS (Ayache et al., 2017). However, studies to date have tended to use measures of only a single modality, usually photographs of faces. PwMS have difficulties in labelling emotions from standardised photographs of facial expressions (e.g. Beatty et al., 1989; Berneiser et al., 2014), and from auditory cues (e.g. Beatty et al., 2003; Kraemar et al., 2013). Few studies have investigated the effects of MS on emotion perception beyond single modality presentations of facial photographs or auditory expressions.

In order to understand the wider implications of emotion perception problems in MS it is important to use multimodal dynamic videos. Such stimuli are more representative of emotion decoding in everyday life, where emotions are seen in context, with dynamic, fleeting and often subtle information from multiple channels. Integration of different modalities occurs early in processing information to provide a multisensory and holistic 
experience of affective information (Gerdes, Wieser \& Alpers, 2014). Multisensory

integration speeds up and improves our ability to understand others' emotions, and so multimodal presentation of emotional information may assist emotion perception for pwMS. Another possibility is that reduced white matter connectivity in MS may result in inefficient multisensory integration, as has been shown for example in schizophrenia (Tseng, Bossong, Modinos, Chen, Mcguire, \& Allen, 2015). This could potentially result in more difficulties in multimodal emotion perception for pwMS. We are not aware of any previous studies which looked at emotion perception from visual, auditory and multimodal tasks in the same sample of MS participants and controls to allow comparison between the tasks, and that is therefore a focus of the current paper.

There are few studies which directly assess multimodal emotion perception in MS. A number of studies have looked at emotion understanding as one aspect of broader theory of mind in MS, using the multimodal video Movie for the Assessment of Social Cognition (MASC). Pöttgen et al. (2013) reported that PwMS performed significantly worse on the 17 emotion recognition questions of the MASC compared to healthy controls, while in contrast Isernia et al. (2019) found no effect of MS. Genova, Cagna, Chiaravalloti, DeLuca \& Lengenfelder (2016) have shown that pwMS score worse than controls on multimodal assessments of another aspect of theory of mind: understanding sarcasm and lies. However, to date there is only one study that we are aware that looked directly at the effects of MS on multimodal emotion perception: Genova and MacDonald (2020) reported in a pilot study that 15 participants with progressive MS were impaired on The Awareness of Social Inference Test - Short Form (TASIT-S). In the current study, as well as unimodal emotion labelling tasks, participants completed the full version of the TASIT multimodal emotion 
perception task. This task uses videos of actors displaying complex dynamic, visual and auditory cues in a realistic context to assess emotion recognition.

Emotion perception tasks require a range of cognitive processes, including rapid attentional processing and executive functions such as working memory (Phillips et al., 2008, Berneiser et al., 2014). These cognitive functions are also impaired in pwMS. It is important to understand the potential relationship between cognitive and emotional skills in MS in order to best target rehabilitation strategies. Previous evidence on the relationship between processing speed, executive function and emotion perception in MS is somewhat mixed. While some studies have found significant associations between facial emotion perception and speed of processing as assessed by the Symbol Digit Modalities Test (Cecchetto et al., 2014, Henry et al., 2009) others have not (e.g.; Dulau et al., 2017). Executive function correlated highly with facial emotion perception in those with MS in some studies (Henry et al., 2009) yet did not correlate at all in others (Cecchetto et al., 2014). Kraemer et al. (2013) reported no association between executive function and basic perception of auditory emotions in pwMS, yet did find a correlation with a task of matching facial to auditory emotions. This indicates the importance of looking (within a single sample) at how cognitive measures might relate to different modalities of emotion perception.

A number of studies indicate that measures of executive function may relate to multimodal measures of theory of mind in MS (see Chalah \& Ayache, 2017 for a review). For example, Genova et al. (2016) reported that the theory of mind tests from the TASIT were related to executive function in a sample of pwMS. However, theory of mind assessments are by their nature cognitively complex, and there is little direct evidence to date on the role of executive function and processing speed in more straightforward multimodal emotion perception in MS. In a pilot study of 15 pwMS, Genova and MacDonald (2020) reported that 
there were not significant correlations between a brief version of the emotion perception task (TASIT-S) and measures of speed or executive function. However, it would be useful to further explore this issue in a larger sample with the full-length multimodal task. Across different studies, discrepancies between these relationships of cognition and emotion perception may be explained by the different (and usually single) measures of emotion perception investigated, or the differing samples of MS participants. In the current study we investigated the relationship between key cognitive constructs (speed, executive function) and emotion perception across three different modalities (facial expressions, auditory intonation, multimodal videos) in the same sample.

Recent reviews argue that emotion perception problems in MS are likely to lead to impaired social functioning (Bora et al., 2016; Cotter et al., 2016). Indeed, problems with emotion perception are related to impaired social function in other neurological conditions such as stroke (Cooper, Phillips, Johnston, Radlak \& Macleod, 2014). However to date there is little published evidence to evaluate this relationship in MS. Phillips, Henry, Scott, Summers, Whyte \& Cook (2011) found correlations between measures of mental state understanding which included an emotional component (Reading the Mind in the Eyes Test, RMET) and quality of life in pwMS, but did not look at whether ability to decode basic emotions from multiple modalities predicted social function. Genova et al. (2020) reported that performance on the RMET in MS was associated with greater fatigue, anxiety and depression. In the current study we directly tested the hypothesis that emotion perception problems in MS predict wider social function using measures of social participation and social network support. Social participation denotes engagement in activities that involve the presence of other people, such as recreational time, socialising, hobbies and work (Ekstrom, Ivanoff, \& Elmstahl, 2008). Here we used the Modified Functional Limitation 
Profile (mFLP; Pollard \& Johnston, 2001) to assess whether pwMS feel that their illness has impacted upon social activities. We also assessed the level of social support that individuals experience using the Lubben Social Network Scale (LSNS-18; Lubben, 1988), separating out support from family and friends. For someone with MS, social and emotional support from their family may be relatively robust to subtle changes in the ability to respond to emotional cues, while on the other hand friendship ties may be more sensitive to these emotional skills.

This study had three aims.

1) To investigate whether the effects of MS on emotion perception are seen in visual, auditory and more naturalistic multimodal representations of emotion.

2) To explore whether emotion recognition performance across these three different tasks are related to speed of processing and executive function in pwMS.

3) To test the hypothesis that difficulties in emotion perception in MS predict restricted social participation and particularly social support from friends.

\section{Methods}

Ethics applications were reviewed and approved by both the local University Psychology Research Committee and under the proportionate review scheme of the UK NHS Research Ethics Committee.

\section{Participants}

MS sample. 53 individuals (38 female) with MS from the local NHS Department of Neurology MS database took part in the study. Most $(n=29)$ had a diagnosis of Relapsing Remitting (RR) MS, 21 a diagnosis of Secondary Progressive MS and three Primary Progressive MS. Sixteen RRMS participants were taking disease-modifying medication. There 
was on average $11.92(S D=7.96)$ years since diagnosis. MS participants met McDonald Criteria for MS diagnosis as determined by a consultant neurologist (McDonald et al., 2001). Participants were excluded if they met any of the following criteria: 1 . Deemed not to have capacity to consent; 2 . Premorbid history of neurological or psychiatric disease (other than MS); 3. Poor understanding of English; 4. Current optic neuritis, or other severe perceptual or motor impairment which would result in an inability to complete the measures, 5 . Less than 18 years old. MS severity was assessed using the Patient Determined Disease Steps (PDDS) questionnaire (Hohol, Orav and Weiner, 1995). In the current sample the mean disease severity rating on the PDDS was $3.44(S D=2.48)$, with a full range of scores from 0 to 8 .

Healthy Control (HC) sample. 31 participants (19 female) were recruited from the general community. Participants were excluded if they met the following criteria: 1 . History or current diagnosis of neurological or psychiatric disease. 2. Poor understanding of English, 3. Aged $<18$.

Procedure

Presentation order of the tasks was counterbalanced to minimise order and fatigue effects. These tasks were part of a wider battery of tasks administered: see Radlak (2014).

\section{Emotion Perception Measures}

Visual Modality- Participants were administered the standardized Facial Expressions of Emotion: Stimuli and Test (FEEST: Young, Perrett, Calder, Sprengelmeyer, \& Ekman, 2002) to assess recognition of basic facial expressions (surprise, disgust, fear, anger, sadness and happiness). There were 60 static, black-and-white pictures of 10 actors showing the emotions. Pictures were displayed in a randomized order on a laptop screen. A single picture was shown for each trial with all six emotion labels visible. Participants clicked on 
the emotion label that best described the facial expression. The dependent variable was percentage accuracy. A number of previous studies have used the Ekman faces presented in the FEEST task to look at the effects of MS on emotion perception, and all have found evidence of impairments (for a review see Chalah \& Ayache, 2017).

Auditory Modality - The Voice Emotion Labeling Subtest (VELS) of the Florida Affect Battery (FAB; Bowers, Blonder, Slomine, \& Heinman, 1996) was used to assess auditory emotion perception. 20 sentences of neutral semantic content were played in a randomized order. The sentences were spoken with an emotional tone in a voice that was either happy, neutral, angry, frightened or sad. Participants indicated which emotion was present by clicking on one of the five options which appeared on screen. The dependent variable was percentage accuracy. Kraemer et al. (2013) used a German version of this task and found that pwMS were impaired in understanding affective prosody.

Multimodal Measure- The Awareness of Social Inference Test (TASIT) Part 1: Emotion Evaluation Test (McDonald et al., 2004) comprised 28 video vignettes of actors, portrayed in everyday settings, expressing one of seven emotional states (happy, surprised, neutral, sad angry, anxious or revolted). The scripts were ambiguous in content. Videos were played on a laptop, and after each one participants indicated which emotion label best described the feelings of the main protagonist. The dependent variable was the percentage accuracy. A recent study indicated that pwMS were impaired compared to healthy controls on a shortened version of this task (Genova \& MacDonald, 2020).

Note that in order to facilitate comparisons between the emotion perception tasks, all of the percentage accuracy scores were converted to standardized ( $z$ ) scores before analysis.

\section{Cognitive Assessment}


Executive function - Measures of verbal fluency are sensitive indicators of cognitive dysfunction in MS (Henry \& Beatty, 2006). In the current study, three trials of letter fluency were used: F, A and S. For each letter, participants said out loud as many words as possible that began with that letter within a one minute time limit. The dependent variable was the number of valid responses averaged across the three letters. For semantic fluency, one trial of category cue 'animals' was used. Participants named as many animals as they could within a one minute time limit. The dependent variable was the number of valid responses. Speed of information processing - The Digit Symbol Coding Task (DSCT) is a processing speed measure (Wechsler, 1997). It is a sensitive marker of cognitive dysfunction in neurological disease (Lezak, Howieson \& Loring, 2004). Participants were asked to mark blank squares on a piece of paper with the appropriate symbol from a list at the top of the page as quickly and accurately as possible for 60 seconds. The dependent variable was the number of symbols filled in correctly. Three participants with MS did not complete this measure.

\section{Social functioning}

Social participation restrictions - The modified Functional Limitations Profile (mFLP, Pollard \& Johnson, 2001) was used to assess participation restriction in pwMS. It differentiates well between social participation restriction and more physical activity limitations (Pollard, Johnston and Dieppe, 2006). Only the five categories established to assess social participation restrictions were administered here: mobility, household management, recreation, social interaction and work. For each item participants were asked whether this restriction applied to them, if so, whether this was due to their MS. The dependent variable was the mean social participation restriction score, with higher scores 
representing more restrictions. This was calculated by averaging percentage scores for each of five participation categories.

Social support - Social support from friends and family was measured using the Lubben Social Network Scale (LSNS: Lubben, Gironda \& Lee, 2002). Participants evaluates the extent of their active social network, how supportive the network is, and the extent to which they can discuss confidential matters. The possible scores for each subscale (family or friends) range from 0 to 30, with higher scores indicating better social networks. Three healthy control participants did not complete this measure.

\section{Analysis}

In order to investigate the effects of MS on the three different measures of emotion perception, an ANOVA tested the between-participants effects of group (MS vs. HC) and within-participants effect of task (visual, auditory, multimodal) on (z-scored) accuracy levels. To address relationships between emotion perception, cognition and social participation, correlational analysis was used. Finally, to test whether emotion perception difficulties in MS predicted variance in social functioning over and above contributions made by disease severity and cognitive function, hierarchical regression analyses were carried out.

\section{Results}

Table 1 presents the descriptive statistics and t-tests for group differences in each of the variables of interest: demographics, emotion perception, cognitive task performance and social functioning.

Effects of MS on different modalities of emotion perception.

To look at the effect of MS on different measures of emotion perception, an ANOVA tested the effects of group (MS vs. HC) and task (visual, auditory, multimodal) on accuracy 
of emotion perception. Descriptive information (percentage accuracy) is shown in Table 1, but note that the actual ANOVA was carried out on standardized $z$ scores. There was an overall effect of MS on emotion perception, $F(1,82)=14.64, p<.001, \eta_{p}{ }^{2}=.151 ;$ pwMS performed more poorly than the HC group. There was no effect of modality on these standardized z scores, $F(2,164)=0.08, \eta_{\mathrm{p}}{ }^{2}=.001$. Note that from the raw data shown in Table 1 accuracy levels on the visual task were lower (78.04\%) than on the auditory $(82.68 \%)$ or multimodal (82.73\%) tasks. The interaction between group and task was not significant, $F(2,164)=1.40, p=.250, \eta_{\mathrm{p}}{ }^{2}=.017$, indicating similar levels of MS-related difficulties on all modalities of emotion perception. Given the similar effects of MS on the different emotion perception tasks, and the moderate intercorrelations between the tasks $(r s>.45)$, a composite total emotion perception score was created for subsequent regression analyses. This score was created by adding together the standard ( $\mathrm{z}$ ) scores for each emotion perception task.

Relationship between emotion perception and cognition.

PwMS performed more poorly than the HC group in terms of fluency and digit symbol scores (see Table 1). There were high correlations between the different cognitive measures ( $r s>.58)$, so these were converted to standard (z) scores and summed to form a total cognition score for subsequent regression analyses. Table 2 shows correlations between emotion perception and cognitive function measures in pwMS. For visual and multimodal emotion perception, there were consistent positive relationships with cognitive test scores. For the auditory task, there were no significant correlations, though the trend was towards positive associations. For the $\mathrm{HC}$ group correlations between emotion perception and cognition were similar but slightly lower, so did not all reach significance. Overall these 
correlations indicate that for pwMS better emotion perception was associated with better cognitive performance.

Emotion perception and social functioning.

As Table 1 indicates, pwMS reported lower levels of social support from friends compared to matched controls, but there was no effect of MS on social support from family. To explore links between emotion perception in MS and social functioning, correlations are reported in Table 3. All modalities of emotion perception were significantly related to perceived levels of social support from friends, but not from family. Better performance on auditory and multimodal emotion perception related to fewer restrictions on social participation. Total emotion perception scores related to both social support from friends and social participation restrictions. For the $\mathrm{HC}$ group, there were was no relationship between emotion perception and social support.

To test whether emotion perception difficulties in MS predicted variance in social functioning over and above contributions made by disease severity and cognitive function, hierarchical regression analyses were carried out (Table 4). In the first analysis, the dependent variable was social support from friends. As a first step disease severity and total cognition score were entered as predictors. This revealed a significant prediction from cognition but not disease severity. In the second step, total emotion perception score was entered. This increased the amount of variance explained, as shown by the significant F(change) value. In the full regression model (including all predictors) emotion perception was the only significant predictor of social support from friends. A separate regression analysis shown in Table 4 (second column) shows that restrictions in social participation were initially predicted by both cognitive performance and disease severity. In the full regression model, emotion perception did not add to the prediction of variance in social 
participation. Disease severity was a strong predictor of restrictions in social participation, with total cognition score also a significant predictor.

\section{Discussion}

Previous studies have indicated that MS can impair emotion perception in visual (Cotter et al., 2016) and auditory (Kraemar et al., 2013) modalities. The current study extended a recent pilot study (Genova and MacDonald, 2020) to indicate that pwMS show equivalent impairments on a more ecologically valid multimodal emotion perception task. On a video-based task which included dynamic visual and auditory information pwMS were less accurate at identifying emotions compared to healthy counterparts. The size of the group difference was similar in the visual and multimodal emotion perception tasks. This indicates that the substantial effects of MS on unimodal tasks such as labelling emotions from photographs extend to decoding the contextualised emotions observed in real social interactions. From a more theoretical perspective, these findings indicate that multisensory integration does not particularly help pwMS to decode emotions, nor do multimodal stimuli appear to be processed more inefficiently in MS due to neural dysconnectivity.

An important point to consider when interpreting these results is that the tasks of emotion perception differed in the range of emotions displayed. While the visual task portrayed the six 'basic' emotions often described in the literature (Ekman, 1999), the auditory task only presented a subset of these emotions. The number of labels to choose from can influence the cognitive load of emotion perception tasks (Phillips et al., 2008). Further, the video stimuli used in the current study showed five of the basic emotions (excluding surprise) and also included neutral portrayals. Given that one of the most common errors in emotion labelling is confusion of fear and surprise (Young et al., 2002) 
this exclusion of surprise may influence performance. Also people may differ in their thresholds in discriminating between 'emotional' and 'neutral', so including a neutral label in one task but not others might influence the outcomes. Finally, there has been considerable critique in recent years of the concept of basic emotions and a call to consider emotions as more individualised experiences and constructs (e.g. Touroutoglou, Lindquist, Dickerson \& Barrett, 2015). All these issues should be considered in designing future research to further understand the effects of MS on different modalities of decoding emotional cues.

Amongst pwMS there were clear associations between poorer emotion perception, slower processing of information, and worse executive function: for visual and multimodal tasks these were of medium effect size. Correlations tended to be lower between auditory emotion perception and cognitive function, and there were also fewer emotional labels to choose from for the auditory task. It would be interesting in future research to ensure that the labels used for visual and auditory emotion tasks were fully matched to see if this factor might explain the lower correlation with cognitive performance in the current sample. The variability in correlations for different emotion perception tasks might explain some of the discrepancies reported in previous studies (Cecchetto et al., 2014; Henry et al., 2009). It is important in future studies to look more broadly at the links between cognitive function and emotion perception problems in MS, using a range of different executive function tasks, for example, to tease apart the possible role of aspects such as inhibition and working memory. While a number of studies have looked at associations between broad cognitive batteries and emotion perception measure in MS, few have looked at a range of both emotion decoding and cognitive measures in a large sample. 
Numerous articles have predicted that difficulty in reading others' emotions in MS will influence wider social engagement (Berneiser et al., 2014; Bora et al., 2016; Cechetto et al., 2014; Genova et al., 2016; Henry et al., 2011; Pottgen et al., 2013) because sensitivity to others' affective states is key to social communication. The current study is the first to examine this link in detail in MS. All three behavioural tasks of emotion perception related to perceived levels of social support from friendships but there was no relationship to family-based social support. This distinction may reflect the different nature of social relationships with family and friends, particularly for people with a chronic illness such as MS. While family support may be relatively robust to subtle changes in the ability to respond to emotional cues, friendship ties may be more sensitive to these social skills. It is important to note, however, that this data is correlational: future studies should explore emotion perception and social support longitudinally in MS to test causal models.

Here we found that emotion perception in MS was related to the levels of social support experienced from friends. It is interesting to note that a similar relationship was not found for the healthy control participants, suggesting that the relationship found in MS does not just reflect more general individual differences. The link between emotion perception and satisfaction with social support from friends is specific to those with MS, and further research should explore whether this extends into other groups who may experience difficulties with social cognition. One potential explanation for this link is that both emotion perception and social support from friends may be influenced by disease severity and cognitive problems. However here hierarchical regression provided evidence against this. It is interesting to note that, in contrast to the correlations with cognitive function, auditory emotion perception related more highly to social function measures than did visual emotion perception. This might reflect the particular importance of picking up nuances of vocal tone 
in social communication with friends, and this issue would be interesting to pursue in more detail in future research.

While the Lubben social support measure is an indicator of the frequency and quality of different types of social contact, social participation restrictions measured by the Functional Limitations Profile ask more directly about the extent to which the ability to engage in social activities has been curtailed by the experience of having MS. Emotion perception was related to social participation restriction, but the association was only significant for the auditory task. This suggests that difficulties in hearing prosodic cues to affective state may be particularly associated with increasing restrictions on social activities. Hierarchical regression analysis indicated that relationships between emotion perception and social participation restriction may reflect shared variance with disease severity and cognitive function. Disease severity was a strong predictor of social participation restrictions attributed to having MS, and this likely reflects the effects of impaired mobility on the ability to engage in social situations.

Given that the effect size of MS on social cognition tasks like emotion perception equals or exceeds the effect size of MS on traditional cognitive tasks (Cotter et al., 2016) there needs to be more awareness amongst clinical teams and pwMS about these issues, and how to assess and deal with them. Understanding emotion perception and social functioning in pwMS can be helpful in identifying patients who may benefit from compensatory strategies, such as encouraging significant others to express emotions and intentions more explicitly. It is also important that future research addresses in more detail the effects of MS on multimodal emotion perception, in more ecologically valid situations. Understanding the nature of any deficits in more realistic situations is important. In addition, the use of multisensory information to enhance emotion perception should be 
investigated, following reports that integrating multiple channels of information can optimise therapeutic approaches in other clinical domains (e.g. Maurage \& Campanella, 2013). Also, a number of environmental and person-centered compensatory strategies can be implemented to better cope with cognitive difficulties that may contribute to socioemotional difficulties, e.g. reducing distractions when conversing or pacing the flow of verbal exchange to account for slowed information processing. Increased knowledge of the nature of any impairment in emotional and social functioning in MS can contribute to improved clinical management and rehabilitation.

Reduced social participation in MS can result in higher levels of depression and isolation (Pakenham, 2006; Kirchner \& Lara, 2011), as well as faster disease progression (Lerdal, Celius, \& Moum, 2009). Addressing factors which might limit social engagement should be a target of rehabilitation programmes, an area of research that is still in its infancy (Kesselring, 2010). The current results indicate that one possible target could be training to improve emotion recognition skills, perhaps amongst other aspects of social cognition. Social cognition skills training has proven successful in improving emotion perception in people with brain injuries (for a review see Struchen, 2014), but has not been widely evaluated to date in pwMS.

\section{Conclusions}

Problems with emotion perception experienced by some pwMS extend to realistic multimodal videos of interpersonal interactions, and relate to cognitive impairments. Our evidence also indicated that these emotion perception difficulties predicted some aspects of social functioning: particularly the level of social support experienced from friends. 


\section{References}

Ayache, S. S., Chalah, M. A., Kuempfel, T., Padberg, F., Lefaucheur, J. P., \& Palm, U. (2017). Facial emotion recognition, theory of mind and empathy in multiple sclerosis. Fortschritte der Neurologie-Psychiatrie, 85, 663-674. doi: 10.1055/s-0043118846

Beatty, W. W., Goodkin, D. E., Weir, W. S., Staton, D., Monson, N., \& Beatty, P. A. (1989). Affective judgments by patients with Parkinson's disease or chronic progressive multiple sclerosis. Bulletin of the Psychonomic Society, 27, 361-364. Retrieved from http://link.springer.com/article/10.3758/BF03334628

Beatty, W. W., Orbelo, D. M., Sorocco, K. H., \& Ross, E. D. (2003). Comprehension of affective prosody in multiple sclerosis. Multiple Sclerosis, 9, 148-153. doi:

\subsection{1/1352458503ms897oa}

Berneiser, J., Wendt, J., Grothe, M., Kessler, C., Hamm, A. O., \& Dressel, A. (2014). Impaired recognition of emotional facial expressions in patients with multiple sclerosis. Multiple Sclerosis and Related Disorders, 3, 482-488. doi: 10.1016/j.msard.2014.02.001

Bora, E., Özakbaş, S., Velakoulis, D., \& Walterfang, M. (2016). Social cognition in multiple sclerosis: a meta-analysis. Neuropsychology Review, 26, 160-172. doi: 10.1007/s11065016-9320-6

Bornhofen, C., \& McDonald, S. (2008). Emotion perception deficits following traumatic brain injury: A review of the evidence and rationale for intervention. Journal of International Neuropsychological Society, 14, 511-525. doi: 10.1017/S1355617708080703

Bowers, D. Blonder, L. X., Slomine, B., \& Heilman, K. M. (1996). Nonverbal Emotional Signals: Patterns of impairments following hemispheric lesions using the Florida Affect Battery. San Francisco CA: American Academy of Neurology. 
Cecchetto, C., Aiello, M., D’Amico, D., Cutuli, D., Cargnelutti, D., Eleopra, R., \& Rumiati, R. I. (2014). Facial and bodily emotion recognition in multiple sclerosis: the role of alexithymia and other characteristics of the disease. Journal of the International Neuropsychological Society, 20, 1004-1014. doi: 10.1017/\$1355617714000939

Chalah, M., \& Ayache, S. (2017). Deficits in social cognition: An unveiled signature of multiple sclerosis. Journal of the International Neuropsychological Society, 23, 266-286. doi:10.1017/S1355617716001156

Compston, A., \& Coles, A. (2002). Multiple Sclerosis. The Lancet, 359 (9313), 1221-1231.doi: 10.1016/S0140-6736(02)08220-X

Cooper, C. L., Phillips, L. H., Johnston, M., Radlak, B., Hamilton, S., \& McLeod, M. J. (2014). Links between emotion perception and social participation restriction following stroke. Brain Injury, 28, 122-126. doi: 10.3109/02699052.2013.848379

Cotter, J., Firth, J., Enzinger, C., Kontopantelis, E., Yung, A. R., Elliott, R., \& Drake, R. J. (2016). Social cognition in multiple sclerosis: a systematic review and meta-analysis. Neurology, 87, 1727-1736. doi: 10.1212/WNL.0000000000003236

Dulau, C., Deloire, M., Diaz, H., Saubusse, A., Charre-Morin, J., Prouteau, A., \& Brochet, B. (2017). Social cognition according to cognitive impairment in different clinical phenotypes of multiple sclerosis. Journal of Neurology, 264, 740-748. doi: $10.1007 / \mathrm{s} 00415-017-8417-z$

Einarsson U, Gottberg K, Von Koch L, Fredrikson S, Ytterberg C, Jin Y. P, ... , \& Holmqvist, L.W. (2006). Cognitive and motor function in people with multiple sclerosis in Stockholm County. Multiple Sclerosis, 12, 340-353. doi: 10.1191/135248506ms1259oa

Ekstrom, H., Ivanoff, S. D., \& Elmstahl, S. (2008). Restriction in social participation and lower life satisfaction among fractured in pain. Results from the population study "Good aging 
in Skane". Archives of Gerentology and Geriatrics, 46, 409- 424. doi:

10.1016/j.archger.2007.06.001

Ekman, P. (1999). Basic emotions. In T. Dalgleish \& T. Power (Eds.), The Handbook of Cognition and Emotion (pp. 45-60). Sussex: John Wiley \& Sons, Ltd.

Genova, H. M., Lancaster, K., Lengenfelder, J., Bober, C. P., DeLuca, J., \& Chiaravalloti, N. D. (2020). Relationship between social cognition and fatigue, depressive symptoms, and anxiety in multiple sclerosis. Journal of Neuropsychology, 14, 213-225.

doi:10.1111/jnp.12185

Genova, H., Cagna, C., Chiaravalloti, N., DeLuca, J., \& Lengenfelder, J. (2016). Dynamic assessment of social cognition in individuals with multiple sclerosis: A pilot study. Journal of the International Neuropsychological Society, 22, 83-88. doi:10.1017/S1355617715001137

Genova, H., \& McDonald, S. (2020). Social cognition in individuals with progressive multiple sclerosis: A pilot study using TASIT-S. Journal of the International Neuropsychological Society, 26, 539-544. doi:10.1017/S1355617719001371

Gerdes, A., Wieser, M. J., \& Alpers, G. W. (2014). Emotional pictures and sounds: a review of multimodal interactions of emotion cues in multiple domains. Frontiers in Psychology, 5, 1351. doi: 10.3389/fpsyg.2014.01351

Henry, J. D., \& Beatty, W. B. (2006). Verbal fluency deficits in multiple sclerosis. Neuropsychologia, 44, 1166-1174. doi: 10.1016/j.neuropsychologia.2005.10.006 Henry, J. D., Phillips, L. H., Beatty, W. W., McDonald, S., Longley, W. A., Joscelyne, A., \& Rendell, P. G. (2009). Evidence for deficits in facial affect recognition and theory of mind in multiple sclerosis. Journal of the International Neuropsychological Society, 15, 277285. doi: $10.1017 /$ S1355617709090195 
Henry, A., Tourbah, A., Chaunu, M. P., Rumbach, L., Montreuil, M., \& Bakchine, S. (2011). Social cognition impairments in relapsing-remitting Multiple Sclerosis. Journal of International Neuropsychological Society, 17, 1122-131. doi:1017/S1355617711001147 Hohol, M. J., Orav, E. J., \& Weiner, H. L. (1995). Disease steps in multiple sclerosis: a simple approach to evaluate disease progression. Neurology, 45, 251-255. doi: 10.1212/WNL.45.2.251

Isernia, S., Baglio, F., d’Arma, A., Groppo, E., Marchett,i A. \& Massaro, D. (2019). Social mind and long-lasting disease: Focus on affective and cognitive theory of mind in Multiple Sclerosis. Frontiers in Psychology, 10, 218. doi: 10.3389/fpsyg.2019.00218

Kesselring, J. (2010). Disease Progression in Multiple Sclerosis I. Impaired Mobility and Its Impact on Limitations of Activities and Social Participation. European Neurological Review, 5, 56-60. doi: 10.17925/enr.2010.05.01.56

Kirchner, T., \& Lara, S. (2011). Stress and depression symptoms in patients with multiple sclerosis: the mediating role of the loss of social functioning. Acta Neurologica Scandinavica, 123, 407-413. doi: 10.1111/j.1600-0404.2010.01422.x

Kraemer, M., Herold, M., Uekermann, J., Kis, B., Daum, I., Wiltfang, J., ..., \& Abdel-Hamid, M. (2013). Perception of affective prosody in patients at an early stage of relapsingremitting multiple sclerosis. Journal of Neuropsychology, 7, 91- 106. doi: 10.1111/j.1748-6653.2012.02037.x

Lerdal, A., Celius, E. G., \& Moum' T. (2009). Perceptions of illness and its development in patients with multiple sclerosis: a prospective cohort study. Journal of Advanced Nursing, 65, 184-192. doi: 10.1111/j.1365-2648.2008.04862.x

Lezak, M. D., Howieson, D. B., \& Loring, D. W. (2004). Neuropsychological Assessment (4th ed.). Oxford: Oxford University Press. 
Lubben, J. E. (1988). Assessing social networks among elderly populations. Family \& Community Health, 11, 42-52.

Lubben, J., Gironda, M. \& Less, A. (2002). Lubben Social Network Scale- Revised. The Behavioral Measurement Letters, 7, 2-11.

Maurage, P., and Campanella, S.(2013). Experimental and clinical usefulness of crossmodal paradigms in psychiatry: an illustration from emotional processing in alcoholdependence. Frontiers in Human Neuroscience, 7, 394. doi:10.3389/fnhum.2013.00394 McDonald, S., \& Flanagan, S., Martin, I., \& Saunders, C. (2004). The ecological validity of TASIT: A test of social perception. Neuropsychological Rehabilitation, 14, 285-302. doi: $10.1080 / 09602010343000237$

McDonald, W. I., Compston, A., Edan, G., Goodkin, D. E., Hartung, H. P., Lublin, F. D., ... , \& Wolinsky, J. S. (2001). Recommended diagnostic criteria for multiple sclerosis: Guidelines from the International Panel on the diagnosis of multiple sclerosis. Annals of Neurology, 50, 121-127. doi: 10.1002/ana.1032

Pakenham, K. I. (2006). Investigation of the coping antecedents to positive outcomes and distress in multiple sclerosis (MS). Psychology and Health, 21, 633-649. doi:

\subsection{1\%2F0269215506cre976oa}

Phillips, L.H., Channon, S., Tunstall, M., Hedenstrom, A \& Lyons, K. (2008). The role of working memory in decoding emotions. Emotion, 8, 184-191. doi: 10.1037/15283542.8.2.184

Phillips, L. H., Henry, J. D., Scott, C., Summers, F., Whyte, M., \& Cook, M. (2011). Specific impairments of emotion perception in multiple sclerosis. Neuropsychology, 25, 131-136. doi:10.1037/a0020752 
Pollard, B., \& Johnston, M. (2001). Problems with the Sickness Impact Profile: a theoretically based analysis and a proposal for a new method of implementation and scoring. Social Science \& Medicine, 52, 921-934. doi: 10.1016/S0277-9536(00)00194-5

Pollard, B., Johnston, M., \& Dieppe, P. (2006). What do osteoarthritis health outcome instruments measure? Impairment, Activity Limitation, or Participation Restriction? Journal of Rheumatology, 33, 757-63.

Pottgen, J., Dziobek, I., Reh, S., Heesen, C., \& Gold, S. M. (2013). Impaired social cognition in multiple sclerosis. Journal of Neurology, Neurosurgery \& Psychiatry, 84, 523-528. doi: 10.1136/jnnp-2012-304157

Radlak, B. (2014). Social cognition in multiple sclerosis: Effects on social participation and quality of life. (Unpublished doctoral dissertation). University of Aberdeen, Aberdeen, UK.

Struchen, M.A. (2014). Social communication interventions (p213-231). In M. Sherer and A.M. Sander (Eds.), Handbook on the Neuropsychology of Traumatic Brain Injury, Clinical Handbooks in Neuropsychology. New York: Springer-Verlag. doi: 10.1007/978-1-49390784-7.

Touroutoglou, A., Lindquist, K. A., Dickerson, B. C., \& Barrett, L. F. (2015). Intrinsic connectivity in the human brain does not reveal networks for 'basic' emotions. Social Cognitive and Affective Neuroscience, 10, 1257-1265. doi: https://doi.org/10.1093/scan/nsv013

Tseng, H.-H., Bossong, M.G., Modinos, G., Chen, K.-M., Mcguire, P., Allen, P. (2015). A systematic review of multisensory cognitive-affective integration in schizophrenia. Neuroscience \& Biobehavioral Reviews, 55, 444-452. doi: 10.1016/j.neubiorev.2015.04.019 
Wechsler, D. (1997). Wechsler Adult Intelligence Scale- III. San Antonio: The Psychological Corporation

Young, A., Perrett, D., Calder, A., Sprengelmeyer, R., \& Ekman, P. (2002). Facial Expressions of Emotion: Stimuli \& Tests [FEEST]. Bury St. Edmunds, UK: Thames Valley Tests. 
Table 1: Descriptive statistics and group differences in background information, cognition and social function measures.

\begin{tabular}{|c|c|c|c|c|c|c|c|c|}
\hline & \multicolumn{2}{|c|}{ pwMS } & \multicolumn{3}{|c|}{$\mathrm{HC}$} & \multirow[b]{2}{*}{ Range } & \multirow[b]{2}{*}{$\mathrm{t}$} & \multirow[b]{2}{*}{$d$} \\
\hline & $M$ & SD & Range & $M$ & SD & & & \\
\hline \multicolumn{9}{|l|}{ Demographics } \\
\hline Age & 50.02 & 10.38 & $27-68$ & 50.16 & 11.45 & $31-71$ & $t(82)=0.06$ & 0.01 \\
\hline Education & 14.61 & 2.94 & $11-23$ & 15.76 & 3.26 & $10-22$ & $t(82)=1.66$ & 0.37 \\
\hline \multicolumn{9}{|l|}{ Emotion perception } \\
\hline Visual & 75.58 & 9.18 & $47-97$ & 82.23 & 6.25 & $72-98$ & $\mathrm{t}(82)=5.68 * *$ & 0.84 \\
\hline Auditory & 80.94 & 11.43 & $40-100$ & 85.64 & 7.04 & $75-100$ & $\mathrm{t}(82)=2.07^{*}$ & 0.49 \\
\hline Multimodal & 79.51 & 12.43 & $53-100$ & 88.23 & 5.28 & $79-96$ & $\mathrm{t}(82)=3.70 * * *$ & 0.91 \\
\hline \multicolumn{9}{|l|}{ Cognition } \\
\hline Letter fluency & 13.10 & 4.33 & $4.33-22.67$ & 16.31 & 4.43 & $9.67-25.33$ & $\mathrm{t}(82)=3.25 * *$ & 0.73 \\
\hline Semantic fluency & 20.30 & 7.00 & $3-36$ & 23.19 & 5.67 & $14-38$ & $t(82)=1.95 t$ & 0.45 \\
\hline Digit symbol & 31.30 & 10.47 & $14-53$ & 43.19 & 8.48 & $26-60$ & $\mathrm{t}(79)=5.33 * * *$ & 1.24 \\
\hline
\end{tabular}

Social function 


$\begin{array}{lcccccccc}\text { Participation restriction }^{\mathrm{a}} & 45.33 & 31.46 & 0-92.22 & - & - & - & - & - \\ \text { Social support (friends) } & 15.55 & 6.70 & 0-30 & 18.43 & 4.61 & 9-29 & \mathrm{t}(79)=2.03 * & 0.50 \\ \text { Social support (family) } & 18.43 & 5.99 & 0-28 & 18.82 & 6.33 & 4-29 & \mathrm{t}(79)=0.27 & 0.06\end{array}$

pwMS: people with Ms. HC: healthy control group.

$\dagger p=.05,^{*} p<.05, * * p<.01, p<.001$

a: Participation restriction cannot be measured in the control group. 
Table 2: Correlations between emotion perception (EP) and cognitive function in the MS group ( $n=53)$ and HC group ( $n=31)$.

Correlations with cognitive function measure in MS group Letter fluency Semantic fluency Digit symbol Total cognition

\begin{tabular}{lllll}
\hline EP modality & & & & \\
Visual & $.404^{* *}$ & $.450 * *$ & $.313^{*}$ & $.460 * *$ \\
Auditory & .210 & $.253+$ & .157 & $.253+$ \\
Multimodal & $.425 * *$ & $.572 * *$ & $.472 * *$ & $.572 * *$ \\
Total EP & $.420 * *$ & $.516 * *$ & $.382 * *$ & $.520 * *$ \\
\hline
\end{tabular}

Correlations with cognitive function measure in $\mathrm{HC}$ group Letter fluency Semantic fluency Digit symbol Total cognition

\section{EP modality}

$\begin{array}{lllll}\text { Visual } & .299 & .309+ & .407 * & .429 * \\ \text { Auditory } & .362 * & .143 & .179 & .302 \\ \text { Multimodal } & .316 \dagger & .217 & .231 & .331 \dagger \\ \text { Total EP } & .435 * & .300 & .373 * & .478 * *\end{array}$

$$
\text { t } p<.10, * p<.05, * * p<.01
$$


Table 3: Correlations between accuracy of emotion perception (EP) and social functioning in the MS group $(n=53)$ and health control $(H C)$ group $(n=31)$.

Correlations with social function measures in MS

$\begin{array}{ccc}\text { Social support } & \text { Social support } & \text { Restrictions in social } \\ \text { Friends } & \text { Family } & \text { participation }\end{array}$

\begin{tabular}{llll}
\hline EP modality & $.285^{*}$ & .061 & -.141 \\
Visual & $.417^{* *}$ & .064 & $-.319 *$ \\
Auditory & $.349^{*}$ & .147 & $-.258 \dagger$ \\
Multimodal & $.427^{* *}$ & .058 & $-.293^{*}$ \\
Total EP & & & \\
\hline
\end{tabular}

Correlations with social function measures in $\mathrm{HC}$

Social support Social support

Friends $\quad$ Family

\begin{tabular}{lll}
\hline EP modality & \\
Visual & -.040 & -.125 \\
Auditory & -.171 & -.159 \\
Multimodal & -.291 & .001 \\
Total EP & -.203 & -.139 \\
\hline
\end{tabular}

$$
+p<.10,^{*} p<.05, * * p<.01 .
$$


Table 4: Regression analyses showing prediction of social functioning variables in MS from disease severity and cognitive performance (Step 1) and in addition emotion perception (EP) performance (Step 2). Note that separate analyses were carried out to predict social support from friends and social participation restrictions.

Social support

Friends
Restrictions in social

participation

\section{Step 1}

Total cognition $(\beta)$

Severity ( $\beta$ )

$$
-.247
$$

.589

$$
F(2,46)=6.72 * *
$$

$$
\mathrm{F}(2,46)=28.67 * *
$$

Step 2

$\begin{array}{lcc}\text { F(change) } & F(1,45)=4.93 * & F(1,45)=0.01 \\ \text { Total cognition }(\beta) & .151 & -.259 * \\ \text { Severity }(\beta) & -.254 & .590 * * \\ \text { Total EP } & .319 * & -.011\end{array}$

Overall model 